\title{
Youth Sexual Development: A Primer for Social Workers
}

\author{
Richard A. Brandon-Friedman, PhD, LCSW, LCAC \\ Indiana University School of Social Work
}

Corresponding Author:

Richard A. Brandon-Friedman (rifriedm@iupui.edu)

Contact Information:

Email: rifriedm@iupui.edu

This is the author's manuscript of the article published in final edited form as:

Brandon-Friedman, R. A. (2019). Youth sexual identity development: A primer for social workers. Social Work, 64(4), 356-364. https://doi.org/10.1093/sw/swz027 


\begin{abstract}
Sexuality and sexual expression are core aspects of most clients' lives, yet most schools of social work fail to cover sexual development, leaving social workers uninformed about this essential area of human development. This is particularly the case when the sexual development of youths is concerned, as youth sexuality is often considered too controversial to explore. Considering a positive approach to youth sexuality that seeks to enhance youths' sexual development and promote their achievement of full sexual and reproductive rights, this article seeks to provide social workers with a resource they can use to enhance their understanding of youth sexual development and its applicability to social work practice. The two main theoretical orientations used to understand sexual identity development are covered as well as the research support for each. Following the explanation of theory is a discussion of how the two theoretical orientations can be integrated to form a more expansive base for the understanding of sexual identity development. A final section provides guidance on how an advanced understanding of youthful sexual identity development can enhance social work practice at the micro, mezzo, and macro levels.
\end{abstract}

KEY WORDS: adolescent sexuality; sexual development; sexual identity; theories of sexuality; youth sexual development 
Youth Sexual Development: A Primer for Social Workers

In the United States, few topics spur more debate than youth sexual development. Built on a neoconservative ideology, the prevailing wisdom in sectors of American society is children need to be "protected" from discussions of sex and sexuality. When discussion does occur, it is often within a negative framework focused on avoidance or within a disease model that teaches youths to fear their bodies and desires (Fortenberry, 2014). Accepting these norms, many schools of social work have resisted efforts to incorporate material on youth sexual orientation, sexual development, sexual identities, or sexual activities into their core curricula (McCave, Shepard, \& Winter, 2014).

Yet sexuality and sexual expression are core aspects of clients' lives. Actions that could be perceived as sexual occur from infancy onward. While it is reasonable to question whether the actions are "sexual" as understood by adults, infants' and toddlers' genital exploration is well acknowledged (Thigpen, 2012). Active exploration into sexual selfhood is less prevalent in middle childhood and then occurs again in late childhood. Twenty-five percent of young adults report thinking about sex "a lot" as 11- to 12-year-olds (Larsson \& Svedin, 2002), whereas sexual minority youths report becoming aware of same-sex attractions at the average age of 9.8 years (Grossman, Foss, \& D’Augelli, 2014). During adolescence, youths’ sexual exploration increases as romantic and sexual relationships become more socially and personally important (Fortenberry, 2014; Tolman \& McClelland, 2011).

Understanding the role of sexuality in youths' lives requires attention to sexual identity development. Although many conflate "sexual identity" and "sexual orientation," sexual identity is a much broader construct that encompasses all personal and social aspects of individuals' lives relating to the domains of sexual orientation, sexual activities, and romantic desires, all of which 
have direct and indirect effects on the sense of self and ability to engage successfully with others, avoid negative sexual outcomes, reach educational and occupational goals, and enter and maintain positive social, romantic, and sexual relationships. Thus, while sexual orientation is an important part of sexual identity, it is only one piece.

Early recognition of sexual identities, expression of sexual desires, and enactment of aspects of sexual identities are beneficial to individuals' physical health, mental health, social integration or isolation, healthy development, psychosocial functioning, later sexual satisfaction, and overall sexual well-being (Harden, 2014; Heywood, Patrick, Smith, \& Pitts, 2015). Yet without adequate psychosocial supports and the ability to advance their sexual knowledge, youths may struggle to attain the intrapsychic sexual maturity necessary to understand these aspects of their lives and make informed decisions. Furthermore, competing sexual messaging can confuse youths as they seek to make sense of their desires within social milieus that range from the sexually explicit to the sexually oppressive. Considering a positive approach to youth sexuality that seeks to enhance youths' sexual development and promote their achievement of full sexual and reproductive rights, this article seeks to better inform social workers about youth sexual development and the applicability of this knowledge to work with youths.

\section{Sexual Identity Development}

Two main theoretical perspectives dominate the literature on sexual identity development: one based in developmental psychology and the other in theories of sexual socialization. Youths are exposed to progressively more sexualized content in the media and popular culture as they age (Gagnon \& Simon, 2005/1973; Wright, 2009). Rather than highlighting risk, media displays of youth sexuality often suggest sex is pleasurable, desirable, and an expected aspect of youths' lives (Tolman \& McClelland, 2011; Wright, 2009). Media presentations and peer interactions 
often emphasize sexual activities, leading youths to believe their peers are engaging in more sexual activity than is actually occurring and subsequently influencing them to engage in sexual activities themselves, thereby perpetuating a repetitive cycle (Miranda-Diaz \& Corcoran, 2012). From a socialization perspective, some suggest there has been a social determination that adolescence is a time for sexual exploration and that youths are prodded in this direction by social norms. In other words, as youths age, they are increasingly sexualized through media and social messaging that provides them with a sense they should be "sexual" and with directives on sexual enactment that are then followed.

Conversely, it has been suggested it is not that youths' sexual interests have been socially instigated, but rather popular media and the availability of open discussions about sexuality provide youths the means through which to understand changes already occurring in their bodies and psyches (Fortenberry, 2014; Tolman \& McClelland, 2011). Within this developmentally based framework, physiological changes in the youths' bodies and an intrapsychic sexual development process have heightened the salience of sexual exploration, and their pursuit of sexualized media is a manifestation of their drive toward sexual discovery. In other words, physiological or intrapsychic development leads to pursuit of sexual information.

Discussions about sexual development are often framed as a dichotomous clash between biological or intrapsychic development and sociosexualization, but sexual development has myriad influences, and both biology and society play a role in how individuals understand themselves as sexual beings. Acknowledging the risk that dividing this discussion into two discrete sections may perpetuate a faulty compartmentalization, this article does so to allow for an easier understanding of the complex sexual development process. The final section discusses 
sexual behaviors and how these can be understood within each of these frameworks and provides recommendations for incorporating youth sexual development into social work practice.

\section{Normative Models of Sexual Identity Development}

Normative models of sexual identity development are grounded in psychoanalytic and ego-identity theories. Freud's five-stage theory of psychosexual development represented the first epigenetic theory of normative sexual development and continues to dominate much research on how individuals' sexual identities develop. Framed within an essentialist paradigm, Freud posited two innate drives—-sex and aggression—-form individuals' psychic cores. As individuals mature, sexual aspects of the psyche proceed through a psychosexual development process that activates various erogenous zones, starting with the oral and ending with the phallic. Through successful navigation of this developmental sequence, individuals learn to manage their sexual impulses and desires.

Building on Freud's work, Erikson developed an eight-stage epigenetic theory of egoidentity development. Of particular importance to sexual identity development are the fifth and sixth stages, Identity versus Role Confusion and Intimacy versus Isolation, which occur from early to mid-adolescence and late adolescence to young adulthood, respectively. During Identity versus Role Confusion, individuals experience an "identity crisis" and must resolve the existential crisis of who they will be as independent individuals no longer reliant on their families for their identities. They seek and form complex social bonds with other youths that help define their sense of selfhood. This is a time of social and personal self-discovery, often including sexual experimentation. The succeeding stage of Intimacy versus Isolation furthers this development as individuals balance a need to sustain an autonomous identity with a desire to 
form lasting interpersonal relationships. As intimacy develops, individuals incorporate their sexuality into these relationships in a reciprocal manner.

Erikson's idea of individual identity development has been adapted to sub-identities such as sexual identity. Demonstrating the early conflation of sexual identity and sexual orientation identity, early models of "sexual identity" development focused on sexual orientation identity among gay men. Cass (1979) and Troiden (1988) developed the first two models of "homosexual identity development," describing the processes of gay men as they begin to recognize they are homosexual, accept it, and then reveal it to others. Although initially developed with gay men, today the models are generally recognized as representing the sexual orientation identity development process for individuals who identify within any category of sexual minority.

Cass's (1979) model contains six stages: Identity Confusion, when individuals begin to connect discussions about homosexuality to themselves; Identity Comparison, when individuals accept they might be homosexual and compare their sexual identities with others' sexual identities; Identity Tolerance, when individuals begin tolerating they may be homosexual; Identity Acceptance, when individuals fully accept their homosexual identity; Identity Pride, when individuals proudly present themselves as a homosexual to others; and Identity Synthesis, when individuals incorporate their homosexual identity into their global identities.

Troiden's (1988) model is similar to Cass's (1979) model but emphasized multiple aspects of sexual identity, the self, which is how individuals conceptualize their own sexual identity; the perceived, which reflects how others judge individuals' sexual identity; and the presented, which entails how individuals present themselves to others. While these aspects may be congruent, they can also vary completely, as some individuals present publicly very 
differently than they themselves feel or present in private. This can include not only sexual orientation, but also sexual desires and activities such as open relationships.

Worthington, Savoy, Dillon, and Vernaglia (2002) proposed the first influential model of heterosexual identity development. The model is based on a two-dimensional exploration of sexual identity: the intrapsychic development of the individual and the individual's social development. This conceptualization resulted in a matrix of five "statuses," named as such to reduce the implication that sexual identity development requires a specific temporal progression.

Individuals in Worthington et al.'s (2002) Unexplored Commitment status have not explored their sexual identity but are committed to one socially assigned to them. Adhering strictly to social messaging, they are often highly critical of others who differ from themselves, basing judgments of others' sexual actions exclusively on social norms. Diffusion represents a lack of self-awareness regarding sexual identity exploration despite an active rejection of social prescriptions regarding sexuality. Individuals in this stage are likely to have chaotic sexual experiences throughout their lives in which their sexual desires, thoughts, and actions are not bound by social norms, but nor are they consciously explored.

Active Exploration characterizes a time when individuals actively explore their sexual identities. While this exploration may be cognitive or behavioral (or both), the defining feature is its intentionality. This exploration must also purposefully go beyond socially prescribed roles or norms and involve conscious introspection in order to represent movement from Unexplored Commitment. This period often coincides with physiological changes such as puberty, but the two are not necessarily temporally symmetrical.

Within Deepening and Commitment, individuals actively and consciously work toward committing to a personalized sexual identity. For some, strong societal messages can lead to a 
strengthened commitment to a concrete sexual identity based on social prescriptions, but there must be some reflective consideration. Worthington et al. (2002) noted that for individuals who identify as sexual or gender minorities (SGMs), movement into this status without active exploration is unlikely as their incongruence with societal norms often necessitates exploration of aspects of sexual identity.

Worthington et al.'s (2002) most advanced stage is Synthesis, which represents the culmination of sexual identity development. Having actively explored their sexual identity, individuals feel confident with their sexual identity and its congruence with other aspects of their global identity. Given the degree of exploration required to achieve this status, the authors suggested that many individuals do not reach this stage but those who do are more likely to be open to others' sexual identities and the variety of ways they can be expressed. Individuals who identify as SGMs may be more likely to reach this stage owing to the complex reflection many undergo when exploring their sexual orientation identities.

\section{Research Support}

Normative theories of sexual development are often criticized for oversimplifying the developmental process and suggesting individuals who do not follow the stages in the prescribed order or do not reach the "end" are abnormal. Worthington et al.'s (2002) model was designed to address this by organizing people per their current status, not in terms of progression. Further, all theories simplify human actions to understand them, so while this criticism is well warranted, the value of these types of theories must also be acknowledged.

Research using Worthington, Navarro, Savoy, and Hampton’s (2008) Measure of Sexual Identity Exploration and Commitment has demonstrated that positive sexual identity development influences sexual health. More advanced levels of sexual identity development 
have been linked with increased sexual self-efficacy, sexual assertiveness, and levels of psychosocial sexual health, as well as improved overall sexual well-being (Muise, Preyde, Maitland, \& Milhausen, 2010; Parent, Talley, Schwartz, \& Hancock, 2015; Worthington et al., 2008). These increases in sexual health and well-being are essential, as both have been shown to affect overall psychosocial health and positive social engagement (Anderson, 2013).

\section{Summary}

Although sexual identity development has not been linked directly to overall well-being, a chain of relationships has been established. Advancement in the sexual identity development process has been connected with overall sexual well-being, which itself has been correlated with positive psychosocial outcomes. Thus, although caution is necessary when linking findings between studies, empirical investigation has demonstrated the value of normative models of sexual identity development and suggests addressing this subpart of youths' identities may be beneficial to their overall well-being. To understand youth sexual development more fully, however, it is necessary to examine other aspects of youths' lives that affect their sexual development.

\section{Models of Sociosexualization}

Most theories that fit under the broad umbrella of sexual socialization are based on the work of Gagnon and Simon (2005/1973). These theories suggest individuals learn to be sexual through a socialization process that "teaches" them what is sexual, how to act sexually, and what is sexually appropriate or inappropriate. Gagnon and Simon's theory of sexual scripting contains three levels: cultural scripts, interpersonal scripts, and intrapsychic scripts. At the cultural level, social, institutional, and familial entities provide youths with messaging about what behaviors are sexual, when actions or thoughts can be understood as sexual, and what sexuality looks like. Cultural scripts do not direct specific behaviors or thoughts, but rather provide broad templates 
regarding sexual roles and relationships. On the interpersonal level, individuals interpret general cultural guidelines and apply them to their own social interactions. Intrapsychic scripts then contain the processing of the desires, memories, and fantasies heretofore identified as sexual.

Altogether, an individual receives direction about what is sexual (cultural scripts), applies this through the enactment of scripts with others (interpersonal scripts), and recognizes doing so entails managing and expressing desires that originated within the individual (intrapsychic scripts) but were only labeled as sexual based on social definitions. Thus, while sexuality may be grounded in biological impetus, theories of sexual socialization suggest that the expression of that impetus is channeled in specific directions via social learning; biology provides the drive, culture provides the where, when, how, and with whom the drive is expressed.

Later theories of sexual socialization emphasize the highly socialized aspects of the development and understanding of sexual identities. Wright (2014) developed a model, 3 AM, focused on acquisition, activation, and application of sexual scripts. In this model, exposure to new sexual material teaches individuals new scripts or possibilities of which they were previously unaware (acquisition), prime scripts that they already knew but may not have actively considered or that are newly learned (activation), and encourage the usage of these scripts (application). This model emphasizes the importance of individuals' social locations, for while an infinite amount of scripts is possible, individuals are exposed to a limited repertoire determined largely by their environment and experiences.

Among youth, the most prominent sources of sexual socialization are peers, family members, and the media (Baxter, 2013; Isaacs, 2012; Wright, 2009). Through interactions with individuals and media presentations, youths begin to understand what others term as sexual, how to interpret their "sexual" thoughts, and how to enact their sexual impulses individually and with 
others. Thus, youths' sexual identities vary considerably as each is socially constructed within a framework unique to that youth's experiences.

\section{Research Support}

Theories of sociosexualization have a significant research base. If sexual identity development was purely a physiological process, the same types of sexual behaviors and beliefs would be expected across demographic categories. Yet research has found differences in sexual behaviors and sexual health outcomes based on sex, gender, race or ethnicity, sexual orientation identity, and the way race or ethnicity and sex or gender intersect (Everett, Schnarrs, Rosario, Garofalo, \& Mustanski, 2014; Zimmer-Gembeck, O’Sullivan, Mastro, \& Hewitt-Stubbs, 2016). Research also indicated youths' psychosocial health, experiences of trauma or neglect, and relationships with adults in their lives significantly affect sexual choices and health outcomes (Cunningham, Martinez, Scott-Sheldon, Carey, \& Carey, 2017; DiIorio, Pluhar, \& Belcher, 2003; Szanto, Lyons, \& Kisiel, 2012), emphasizing the importance of social experiences.

Further support is provided through the examination of sexual interaction patterns among two subpopulations of youths. Youth involved with the child welfare system have disproportionately high levels of engagement in sexual risk behaviors and negative sexual health outcomes (Winter, Brandon-Friedman, \& Ely, 2016). Examining the social environment of these youths reveals possible factors: the youths have experienced trauma, and many have come from disruptive family systems, had dysfunctional sexual and romantic relationships modeled for them, and lacked access to sexual education. Even within the foster care system, access to positive sexual supports are often lacking (Aparicio, Pecukonis, \& O’Neale, 2015; Winter et al., 2016). As they develop, the youth engage in the behaviors they have had modeled for them, even 
if such actions may not be the healthiest (Brandon-Friedman, Kinney, Pierce, \& Fortenberry, 2017).

Another population of note is youths who engage in same-sex sexual interactions. Formal sexual education is notoriously inattentive to these youths' sexual educational needs, leading many to use pornography as their primary source of sexual education (Arrington-Sanders et al., 2015). Pornography is neither a realistic portrayal of the nuances of sexual interactions nor a viable source of instruction on safe and prosocial methods of sexual engagement and has been shown to lead to unrealistic sexual beliefs, yet these youths' actions often mirror what they see (Owens, Behun, Manning, \& Reid, 2012). The lack of prosocial sources of sexual education likely contributes to the high rates of negative sexual health outcomes in this population (Arrington-Sanders et al., 2015). In other words, these youth are using what is available to explore how to enact aspects of their sexual identity within their lives, fulfilling a need that might otherwise go unmet despite the concerns that can be raised about their influences.

\section{Summary}

Examined through a lens of sociosexualization, youths' social positions and experiences affect what sexual scripts they acquire, which scripts are activated, and how they are applied. During social interactions, youth continually reassess and revise their personal scripts, making adjustments based on what they found to be effective, pleasurable, or beneficial and discarding those that were experienced negatively or were inconsequential to them. These scripts are then integrated within other aspects of their sexual and overall identities, and through this process, the individuals create their unique sexual identities. 


\section{Integrative Youth Sexual Development}

Integrating normative models of sexual development and models of sociosexualization leads to a sequence in which physiological changes in youths' bodies paired with ego identity development prompts youths to seek to understand themselves and their relationships with others in new ways, including physically. Youths' bodies become more sexually developed, and sexuality plays a bigger role in their physiology. Encouraged by social norms, youths seek out more intimate interpersonal relationships and explore their sexuality within these relationships. Those who identify within the heteronormative narrative are able to base their interactions on predominant social norms, whereas those who identify differently or are more sexually experimental go through an additional intra- and interpersonal process during which they further explore their sexual identity. This process entails further sociosexualization as they seek models with which they identify. Even though this growth is initially foregrounded during youthhood, the process continues throughout the lifespan as individuals' sexual physiology changes along with messaging regarding what forms of sexual expression are socially sanctioned.

For example, youths who identify as sexual minorities often come out during early to mid-adolescence. As parents struggle with this new knowledge, they often suggest their child has been influenced by other individuals who identify as sexual minorities they know or by open discussions about sexual minorities in popular media. This suggestion confounds multiple areas of sexual development, and parents need to be educated about what is really happening.

Youth are not influenced to become a sexual minority. Rather, during this time of selfexploration, they may seek out and discover sexual scripts not previously known. During later childhood, intrapsychic development leads youths to become more attuned to their sameness or difference from others, and when they look back, sexual minorities report recognizing that this is 
the time they were different from peers despite not being able to label what that difference was (Martos, Nezhad, \& Meyer, 2015). This difference becomes more pronounced during adolescence, when they begin to seek more intimate relationships and may find themselves feeling further alienated from what their peers appear to be experiencing. Trying to understand these feelings, they use their increasing independence from their families to seek answers. Through discussion with peers and access to media, they discover others whose sexual scripts allow for same-sex intimacy. They can then explore the applicability of these scripts to their lives, determining if they fit better than the previous scripts. Thus, it was not exposure to other sexual minorities that led them to identify as such themselves, but rather this exposure opened new sexual possibilities of which they were previously unaware and which they found germane to themselves. Further emphasizing the social aspects, while the intrapsychic milestone events are similar across demographic groups, the ages at which they occur vary by race, ethnicity, gender, and age cohort (Martos et al., 2015). Educating parents about these coexisting influences will both help them to understand their child and counteract a harmful narrative about sexual minorities influencing others' sexual orientation.

\section{Application to Social Work Practice}

Difficulties arise when individuals fail to recognize the overall significance of the sexual identity development process. Judgments about the propriety or impropriety of others' sexual actions are generally made from viewers' vantage points, rather than after an exploration of the individuals' views. Some people are condemned, even by social workers, for sexual actions without deeper considerations of the nexus of the rules governing those interactions or attempts to problematize such strictures. While some sexual interactions, such as rape, necessitate universal condemnation, failure to examine the social basis for sexual condemnation can be 
detrimental to the lives of those with whom social workers engage (Brandon-Friedman, 2017; McCave et al., 2014). With their person-in-environment focus, social workers are well situated to explore the sexual development process with the youth with whom they work and to assist others with enhancing their understanding of aspects of others' sexual identities. In their interactions with clients, social workers are trained to examine clients' lives on the micro, mezzo, and macro level and to consider the interplay among these systems.

On a micro level, social workers can assist with both intrapsychic and interpersonal sexual identity development. Many individuals need assistance understanding the interplay of their sexual beliefs, desires, and actions. This may be particularly important for youths who identify as sexual minorities, as many not only cope with adversarial social environments and internalized homonegativity, but also with familial struggles related to acceptance of their sexual orientation. Youth from conservative religious backgrounds may struggle to balance what they were taught regarding sex and sexuality with their own desires and perceptions of peers' actions. Youth who have experienced sexual trauma may have difficulty understanding themselves as self-determining sexual beings and coming to terms with how their traumatic experiences affect both their intrapsychic sexual identity development and interpersonal sexual interactions. In these instances, the ability to help youths to understand how their experiences have affected their sexual development and influenced their sexual scripting as well as an awareness of what periods of intrapsychic or interpersonal sexual development were most affected will be essential.

Alternatively, a social worker working with youth engaging in unsafe sexual behaviors should not shame the youth but examine such behaviors in a sex-positive manner. Of course, social workers must also be attuned to the dangers of some sexual actions, such as autoerotic asphyxiation (intentional restriction of oxygen flow to enhance sexual pleasure), and address 
them accordingly. Interventions must balance client self-determination with safety; social workers should educate the youths on the dangers and encourage safer means of sexual expression and pleasure-seeking while taking care to not reproach the youths. Actions that humiliate the youth not only are ineffective at changing behaviors but contribute to sexual shame and a reduced willingness to discuss this aspect of their identity.

Rather than viewing such actions as behavioral problems, the social worker should explore what scripts the youth are enacting, where they learned them, what role such actions are playing in the youths' lives, and how the youths' actions are influencing their other sexual and romantic relationships. Further exploration could focus on where the youths' sexual development is currently and how their sexual decision making may be influenced by their sexual development level. This ensures the youths' sexual identity and actions are evaluated holistically, not as isolated concerns.

Expanding further into the family, social workers can assist family members to understand and come to terms with how the sexual identities or interactions of family members affect them. Research has shown that family culture not only influences sexual expression but also affects how adults interpret and label youths' behaviors (Thigpen, 2012). Families often must address concerns such as when youths identify as sexual minorities; pregnancy during adolescence; sexual assault; sexual infidelity; or family members' having a sexual fetish or desire that others find offensive. In such cases, social workers must be knowledgeable of both intrapsychic and sociosexual models of development, as each affect how the individuals understand themselves, understand others' sexual messaging and actions, and enact their own sexuality. Each family members' experiences will have varied not only owing to their own personality and belief system, but also based on their social exposure to sexual messaging. 
Navigating the divides between individuals' actions and beliefs and those of their family members will require attention to each of these aspects of their lives.

Mezzo-level interventions should focus on changing attitudes toward groups of individuals whose sexual actions differ from the majority. Working to reduce stigma or expand service provision to those parenting adolescents, those who identify as asexual, those engaged in consensual nonmonogamy, or those who are more sexually permissive. Changing attitudes in these circumstances require social workers to promote a sex-positive or nonjudgmental environment that accepts others' sexual decisions even if they differ from one's own.

On a macro level, social workers attuned to youth sexual development can better advocate for their clients and other youths. The predominant narrative related to youth sexual expression is one of risk and fear, yet there is nothing inherently risky nor anything to fear from youths' healthy sexual expression. Challenging the predominant narrative requires social workers to educate others about youth sexual development, and discussing youth sexuality in an informed and positive manner will help to counteract the prevailing social messaging.

At a personal level, social workers should adhere to recent calls for more integration of sexuality into social work. Social workers' views on sexuality are socially constructed, just like others' views. With their focus on social justice and freedom from oppression, though, they are ethically charged with counteracting mainstream social beliefs and actions when those actions serve to oppress or discriminate against individuals, even when it comes to topics related to sexuality (Brandon-Friedman, 2017; McCave et al., 2014). This essential process can only occur through self-reflection and honest appraisal of themselves and their beliefs, both of which begin with education and introspection. Moving forward, social workers need to focus in on this 
critical area of human development as failure to do so could lead to social workers' unexamined attitudes toward aspects of others' sexual identity, hampering their work with those individuals.

Finally, social work educators must confront institutional biases against discussing sexuality in all its variations. Social workers are taught to ask clients about and address trauma, substance use or abuse, and histories of violence and illegal actions, yet the messaging they receive is often that individuals' sexual actions are too intimate to explore. This promotes a narrative of sexual shame and denies their ability to address this influential area of clients' lives; alternatively, an educational environment that allows open discussion of sexuality provides social work students with the skills necessary to examine their clients' interpersonal lives holistically.

Social workers can begin to advance the cause of achieving sexual justice for youth. Sexuality forms a core part of youths' self-concept, especially during adolescence and young adulthood. By shaming youth or not providing them with the information necessary for healthy sexual development, youths' right to positive sexual selfhood is impeded. Hindering this essential aspect of youths' lives can affect many other areas of their development and overall well-being. If they are instead taught to explore this aspect of themselves in a positive way and to the same degree as they do areas such as hobbies and other forms of peer relationships, they will be able to fully integrate their sexuality into their sense of self better, which research suggests will benefit all areas of their lives. Assisting in this process is not only good for the youth with whom a social worker may engage, but for youth as a whole. 


\section{References}

Anderson, R. M. (2013). Positive sexuality and its impact on overall well-being. Bundesgesundheitsblatt Gesundheitsforschung Gesundheitsschutz, 56, 208-214.

Aparicio, E., Pecukonis, E. V., \& O’Neale, S. (2015). “The love that I was missing”: Exploring the lived experience of motherhood among teen mothers in foster care. Children \& Youth Services Review, 51, 44-54.

Arrington-Sanders, R., Harper, G. W., Morgan, A., Ogunbajo, A., Trent, M., \& Fortenberry, J. D. (2015). The role of sexually explicit material in the sexual development of same-sexattracted Black adolescent males. Archives of Sexual Behavior, 44, 597-608.

Baxter, V. (2013). Early adolescent sexual knowledge, values, and beliefs: The role of mass media, peers, parent communication, and religiousity. (Doctoral dissertation). Retrieved from https://twu-ir.tdl.org/handle/11274/764

Brandon-Friedman, R. A. (2017). Power, deviance, stigma, and control: A sociological reconceptualization of sexuality within social work services. Journal of Sociology and Social Welfare, 44(1), 141-167.

Brandon-Friedman, R. A., Kinney, M. K., Pierce, B., \& Fortenberry, J. D. (2017). Former foster youths' perceptions of their acquisition of sexual health information while in foster care. Poster session presented at the 21st Annual Conference of the Social for Social Work and Research, New Orleans.

Cass, V. C. (1979). Homosexual identity formation: A theoretical model. Journal of Homosexuality, 4, 219-235. 
Cunningham, K., Martinez, D. A., Scott-Sheldon, L.A.J., Carey, K. B., \& Carey, M. P. (2017). Alcohol use and sexual risk behaviors among adolescents with psychiatric disorders: A systematic review and meta-analysis. Journal of Child \& Adolescent Substance Abuse, $26,353-366$.

DiIorio, C., Pluhar, E., \& Belcher, L. (2003). Parent-child communication about sexuality: A review of the literature from 1980-2002. Journal of HIV/AIDS Prevention \& Education for Adolescents \& Children, 5(3-4), 7-32.

Everett, B. G., Schnarrs, P. W., Rosario, M., Garofalo, R., \& Mustanski, B. S. (2014). Sexual orientation disparities in sexually transmitted infection risk behaviors and risk determinants among sexually active adolescent males: Results from a school-based sample. American Journal of Public Health, 104, 1107-1112.

Fortenberry, J. D. (2014). Sexual learning, sexual experience, and healthy adolescent sex. New Directions for Child \& Adolescent Development, 144(Summer), 71-86.

Gagnon, J. H., \& Simon, W. (2005/1973). Sexual conduct: The social sources of human sexuality (2nd ed.). New Brunswick, NJ: Aldine Transaction.

Grossman, A. H., Foss, A. H., \& D’Augelli, A. R. (2014). Puberty: Maturation, timing and adjustment, and sexual identity developmental milestones among lesbian, gay, and bisexual youth. Journal of LGBT Youth, 11, 107-124.

Harden, K. P. (2014). A sex-positive framework for research on adolescent sexuality. Perspectives on Psychological Science, 9, 455-469.

Heywood, W., Patrick, K., Smith, A. M., \& Pitts, M. K. (2015). Associations between early first sexual intercourse and later sexual and reproductive outcomes: A systematic review of population-based data. Archives of Sexual Behavior, 44, 531-569. 
Isaacs, A. M. (2012). Let's talk about sex: How family communication patterns and family sexual communication impact adolescents' and emerging adults' sexual outcomes. (Doctoral dissertation). Retrieved from https://conservancy.umn.edu/handle/11299/137205

Larsson, I., \& Svedin, C.-G. (2002). Sexual experiences in childhood: Young adults' recollections. Archives of Sexual Behavior, 31, 263-273.

Martos, A. J., Nezhad, S., \& Meyer, I. H. (2015). Variations in sexual identity milestones among lesbians, gay men, and bisexuals. Sexuality Research and Social Policy, 12(1), 24-33.

McCave, E., Shepard, B., \& Winter, V. R. (2014). Human sexuality as a critical subfield in social work. Advances in Social Work, 15, 409-427.

Miranda-Diaz, M., \& Corcoran, K. (2012). "All my friends are doing it": The impact of the perception of peer sexuality on adolescents' intent to have sex. Journal of EvidenceBased Social Work, 9, 260-264.

Muise, A., Preyde, M., Maitland, S. B., \& Milhausen, R. R. (2010). Sexual identity and sexual well-being in female heterosexual university students. Archives of Sexual Behavior, 39, 915-925.

Owens, E. W., Behun, R. J., Manning, J. C., \& Reid, R. C. (2012). The impact of Internet pornography on adolescents: A review of the research. Sexual Addiction \& Compulsivity, $19,99-122$.

Parent, M. C., Talley, A. E., Schwartz, E. N., \& Hancock, D. W. (2015). I want your sex: The role of sexual exploration in fostering positive sexual self-concepts for heterosexual and sexual minority women. Psychology of Sexual Orientation and Gender Diversity, 2, 199204. 
Szanto, L., Lyons, J. S., \& Kisiel, C. (2012). Childhood trauma experience and the expression of problematic sexual behavior in children and adolescents in state custody. Residential Treatment for Children \& Youth, 29, 231-249.

Thigpen, J. W. (2012). Childhood sexuality: Exploring culture as context. In L. M. Carpenter \& J. D. DeLamater (Eds.), Sex for life: From virginity to Viagra, how sexuality changes throughout our lives (pp. 45-69). New York: New York University Press.

Tolman, D. L., \& McClelland, S. I. (2011). Normative sexuality development in adolescence: A decade in review, 2000-2009. Journal of Research on Adolescence, 21(1), 242-255.

Troiden, R. R. (1988). Gay and lesbian identity: A sociological analysis. Dix Hills, NY: General Hall.

Winter, V. R., Brandon-Friedman, R. A., \& Ely, G. E. (2016). Sexual health behaviors and outcomes among current and former foster youth: A review of the literature. Children and Youth Services Review, 64(1), 1-14.

Worthington, R. L., Navarro, R. L., Savoy, H. B., \& Hampton, D. (2008). Development, reliability, and validity of the Measure of Sexual Identity Exploration and Commitment (MoSIEC). Developmental Psychology, 44(1), 22-33.

Worthington, R. L., Savoy, H. B., Dillon, F. R., \& Vernaglia, E. R. (2002). Heterosexual identity development: A multidimensional model of individual and social identity. Counseling Psychologist, 30, 496-531.

Wright, P. J. (2009). Sexual socialization messages in mainstream entertainment mass media: A review and synthesis. Sexuality \& Culture, 13, 181-200.

Wright, P. J. (2014). Pornography and the sexual socialization of children: Current knowledge and a theoretical future. Journal of Children and Media, 8, 305-312. 
Zimmer-Gembeck, M. J., O’Sullivan, L. F., Mastro, S., \& Hewitt-Stubbs, G. (2016). Adolescent sexuality: Current directions in research, health, and risk reduction. In M. K. Holt \& A. E. Grills (Eds.), Critical issues in school-based mental health: Evidence-based research, practice, and interventions (pp. 93-104). New York: Routledge.

Richard A. Brandon-Friedman, PhD, LCSW, is assistant professor, School of Social Work, Indiana University, 902 West New York Street, ES 4138, Indianapolis, IN 46202; e-mail: rifriedm@iupui.edu. 